\title{
SPECT/CT Lymphoscintigraphy to Locate the Sentinel Lymph Node in Patients with Melanoma
}

\author{
Roger F. Uren \\ Nuclear Medicine and Diagnostic Ultrasound, RPAH Medical Centre and The Discipline of Medicine, \\ University of Sydney, Sydney, NSW, Australia
}

The major strength of nuclear medicine imaging has always been its high functional resolution, and a good example of this is lymphoscintigraphy using radiocolloids to map the location of the lymph node receiving direct lymph drainage from a solid tumor site. Using this technique the one or two sentinel nodes of the 1,000 or more nodes in the patient are radiolabelled and clearly visible on lymphoscintigraphy. ${ }^{1}$ This same technique, however, also nicely illustrates a limitation of nuclear medicine images: their low anatomical resolution. The surface location of these sentinel nodes (SN) can be marked and we can measure the depth of the $\mathrm{SN}$ from the skin, but the planar nuclear medicine images provide no anatomical information.

Nuclear medicine images can be improved using single photon emission computed tomography (SPECT), which displays tomographic slices of the distribution of tracer in the patient. SPECT was developed in the late 1960s by Kuhl and Edwards in Philadelphia before computed tomography (CT) scans were developed. ${ }^{2}$ SPECT images were better than planar scans as there was improved image contrast of pathological lesions, but anatomical data in these images was still limited; when using SPECT scans in lymphoscintigraphy it is almost nonexistent.

The limitations of this low anatomical resolution in nuclear medicine images, whether planar or tomographic, is dramatically illustrated in the case of ${ }^{18} \mathrm{~F}$-flourodeoxyglucose (FDG) positron emission tomography (PET) scanning. This method was developed in the 1970s and, although of great interest to nuclear medicine physicians as it displayed the glycolytic rate of tumors, it had little impact on clinical practice as clinicians had no confidence

(c) Society of Surgical Oncology 2009

Published Online: 11 April 2009

R. F. Uren

e-mail: ruren@mail.usyd.edu.au in the information. ${ }^{3}$ Very little anatomical data was present even in the three-dimensional (3D) tomographic PET displays. It was not until hybrid imaging devices were produced that allowed PET/CT scans to be performed without moving the patient that the situation changed. ${ }^{4}$

PET/CT allowed precise image fusion of the PET tomographic slices with the corresponding CT anatomic slices with the PET functional data displayed in color against the grey scale background of the CT image. Immediately clinicians could appreciate the importance of the information in these images and PET/CT has now assumed a major role in the staging of solid tumors and in the detection of recurrence. These scans can also be used to monitor the effectiveness of chemotherapy regimens by directly measuring the metabolic activity of the tumor.

It is already clear that the equivalent technology for single photon emitting radionuclides, SPECT/CT, is going to have a similar impact on the clinical usefulness of these tracers. SPECT/CT as part of lymphoscintigraphy is an excellent example of the fusion of the high functional resolution of nuclear medicine images (the radiolabelled $\mathrm{SN})$ with the high anatomical resolution of CT. The technology shows precisely which node is the $\mathrm{SN}$, and its detailed anatomical position can be described.

Several studies have shown that, in patients with melanoma, SPECT/CT provides advantages over planar imaging. These include higher sensitivity for SN detection, detection of SNs in new node fields, improved detection of SNs when near the injection site, detection of deep pelvic SNs in patients with leg melanomas, and improved surgical location by providing exact anatomical location. ${ }^{5-7}$ The study in this issue by van der Ploeg and colleagues, in a group of patients with melanoma with a planar study that was difficult to interpret, an unusual drainage pattern or no drainage to a SN, confirmed increased sensitivity for detecting the $\mathrm{SN}$ and also improved anatomical location 
that altered the surgical approach in $30 \%$ of patients involving a different incision, an incision at another site or an extra incision. ${ }^{8}$ The use of 3D volume-rendered images should enhance the presentation of this data for the operating surgeon.

It is known that the skin can also drain through the body wall to deeply placed SNs in some patients, and SPECT/CT will clearly aid in the surgical location of such nodes. ${ }^{9}$ This pattern most often occurs from the skin of the posterior loin through the posterior body wall to retroperitoneal or paravertebral SNs, and a recent patient of The Sydney Melanoma Unit who showed this drainage to a paravertebral node had this node successfully removed using the information obtained from his SPECT/CT lymphoscintigraphy. It is for this reason that SPECT/CT is likely to be especially useful for imaging the SNs in patients with tumors of the internal organs, though technical difficulties need to be overcome for the problem of injecting the radiocolloid to allow imaging prior to surgery.

One issue that is becoming more often discussed in the diagnostic imaging literature is the dosimetry of these procedures, especially when hybrid devices are used. The key information that the CT image brings to the SPECT/CT interpretation is the precise anatomical location of the functional index lesion seen on the nuclear medicine SPECT image. A high-dose CT is not required to give this location and I would encourage all using SPECT/CT hybrid imaging to use the lowest $\mathrm{CT}$ dose possible in each patient. A high-dose diagnostic CT is simply not needed to get the benefits of SPECT/CT, as it is the precise location of the "hot spot" that is the vital data. This comment applies equally to PET/CT. There will be occasions when a follow-up high-dose diagnostic CT is needed, but they will be few and quite rare when using SPECT/CT as part of lymphoscintigraphy in patients with melanoma.

As a final comment it is important to remember that SPECT/CT alone cannot replace the vital information obtained during dynamic lymphoscintigraphy in patients with melanoma. The identification of the individual lymphatic collectors reaching the $\mathrm{SN}$ on such images is the most accurate method of designating a $\mathrm{SN}$ versus a secondtier node that subsequently receives the radiocolloid. Other useful information that is present on dynamic imaging and not SPECT/CT is the dynamic arrival time of the tracer in the $\mathrm{SN}$, and the number of lymphatic collectors leaving the melanoma site, which is a guide to the number of expected
SNs and the lymph node fields to which the collectors drain. The relative intensity of the nodes is also best illustrated on planar scans. Although a second-tier node may appear well radiolabelled on SPECT/CT, it should not be designated a SN and such a node should not be removed as part of a SN biopsy procedure. To do so would negate the important benefit of SN biopsy, that is, unprecedented nodal staging with minimal morbidity.

SPECT/CT as part of lymphoscintigraphy in patients with melanoma to locate the SN prior to surgical removal is the future, and is here with clear benefits in many patients. We must not abandon dynamic lymphoscintigraphy, however, as we would then be in grave danger of "throwing the baby out with the bathwater" and compromising the great benefits in lymph node staging that have already been achieved using this technology.

\section{REFERENCES}

1. Morton DL, Wen D-R, Wong JH, et al. Technical details of intraoperative lymphatic mapping for early stage melanoma. Arch Surg. 1992;127:392-9.

2. Kuhl DE, Edwards RQ. The Mark 3 scanner: a compact device for multiple-view and section scanning of the brain. Radiology. 1970; 96:563-70.

3. Ter-Pogossian MM, Phelps ME, Hoffman EJ, Mullani NA. A positron-emission transaxial tomograph for nuclear imaging (PETT). Radiology. 1975;114:89-98.

4. Beyer T, Townsend DW, Brun T, Kinahan PE, Charron M, Roddy $\mathrm{R}$, et al. A combined PET/CT scanner for clinical oncology. J Nucl Med. 2000;41:1369-79.

5. Even-Sapir E, Lerman H, Lievshitz G, Khafif A, Fliss DM, Schwartz A, et al. Lymphoscintigraphy for sentinel node mapping using a hybrid SPECT/CT system. J Nucl Med. 2003;44:1413-20.

6. Kretschmer L, Altenvoerde G, Meller J, Zutt M, Funke M, Neumann C, et al. Dynamic lymphoscintigraphy and image fusion of SPECT and pelvic CT-scans allow mapping of aberrant pelvic sentinel lymph nodes in malignant melanoma. Eur $J$ Cancer. 2003;39:175-83.

7. van der Ploeg IMC, Valdes Olmos RA, Nieweg OE, Rutgers EJT, Kroon BBR, Hoefnagel CA. The additional value of SPECT/CT in lymphatic mapping in breast cancer and melanoma. J Nucl Med. 2007;48:1756-60.

8. Van der Ploeg IMC, Valdés Olmos RA, Kroon BBR, Wouters MWJM, van den Brekel MWM, Vogel WV, et al. The yield of SPECT/CT for anatomical lymphatic mapping in patients with melanoma. Ann Surg Oncol. 2009. DOI:10.1245/s10434-0090339-2.

9. Uren RF, Howman-Giles RB, Shaw HM, Thompson JF, McCarthy WH. Lymphoscintigraphy in high risk melanoma of the trunk: predicting draining node groups, defining lymphatic channels and locating the sentinel node. J Nucl Med. 34:1435-40,1993. 